\title{
TRANSITIONAL SURROGATES
}

\author{
Pierre Borgnat, Patrick Flandrin \\ Laboratoire de Physique (UMR 5672 CNRS) \\ École Normale Supérieure de Lyon \\ 46 allée d'Italie 69364 Lyon Cedex 07 France
}

\author{
André Ferrari, Cédric Richard \\ Laboratoire Hippolyte Fizeau CNRS-OCA \\ Université de Nice Sophia-Antipolis \\ Parc Valrose 06108 Nice Cedex 2 France
}

\begin{abstract}
While an exact stationarization of a process with a given spectrum magnitude can be obtained via a complete randomization of the spectrum phase ("surrogates" technique), we propose here a softened version in which the degree of stationarization can be controlled by a perturbation of the actual phase. A basic theory for such "transitional surrogates" is first discussed, with emphasis on two effective constructions based on either white Gaussian noise or random walks. Some typical examples are considered for illustration, and performance evaluations are provided for supporting the usefulness of the approach in the context of stationarity testing.
\end{abstract}

Index Terms - Surrogates, stationarity test, random phase

\section{CLASSICAL SURROGATES AS STATIONARIZERS}

\subsection{Rationale}

Initially proposed in the context of nonlinear dynamics [1], the idea of "surrogate data" has recently been pushed forward in nonstationary signal analysis for a purpose of stationarization [2]. In the classical formulation, surrogates are obtained from a given observed signal by keeping unchanged its spectrum magnitude while replacing its spectrum phase by a random sequence. The rationale is that, since the spectrum phase conveys the information about the organization of spectral components in the time domain, a random phase is expected to destroy in the original signal those nonstationarities (if any) that would end up in variations of local spectral properties, while exactly preserving the same global spectrum that is supposed to be time-invariant in the case of a stationary process. This intuition can be made precise, and it has been proved that, under specific assumptions on the phase distribution, surrogates obtained this way are indeed second-order [2] and even strict-sense [3] stationary, thus paving the way for designing stationarity tests based on some comparison between local and global spectra, in which surrogates allow for a statistical characterization of the null hypothesis of stationarity.

\subsection{Limitations}

Testing for stationarity via surrogates has been discussed in [2]. Two companion approaches have been proposed: the first one makes use of (local vs. global) distances whose statistical properties attached to the null hypothesis are derived from simulations run with a number of independent surrogates; the second one considers such an ensemble of stationarized surrogates as a learning set for stationarity, making possible to use the powerful machinery of machine learning methods such as one-class SVMs.

Whereas this proved effective for evidencing nonstationarities in various scenarii, the tests happen to be very sensitive. For instance, when applied to realizations of actual stationary (e.g., AR) processes, the rejection rate of the null hypothesis turns out to be slightly higher than the prescribed confidence level [2]. In a related way, one key point of the approach is to encompass in a common (time-frequency) framework stochastic and deterministic situations, stationarity referring to pure tones in the latter case. In this case too (see Fig. 7 in [2]), surrogates cannot really reproduce the supposed stationarity of the observation. This is a natural outcome of the intrinsically stochastic generation of surrogates, but this makes again the test somehow pessimistic.

The observation of such remaining limitations in the use of "classical" surrogates for testing stationarity prompts to think about related, possibly more versatile constructions: this is the purpose of this study.

\section{FROM CLASSICAL TO TRANSITIONAL SURROGATES}

Let us consider an observed signal $\{x[n], n \in \mathbb{Z}\}$ whose (deterministic) realization has a discrete-frequency Fourier transform

$$
X[f]=A_{X}[f] \exp \left\{i \Psi_{X}[f]\right\}, f \in \mathbb{Z} .
$$

According to the classical definition [1], associating a surrogate to such an observation would amount to throw away the actual spectrum phase $\Psi_{X}[f]$ and to replace it by some i.i.d. sequence. ${ }^{1}$ This is effective in terms of stationarization $[2,3]$ but somehow binary in the sense that, after the phase replacement, a possibly nonstationary signal is actually turned into an exactly stationary one, without any consideration about the fact that, from an operational perspective, stationarity has to be understood in a relative sense (see [2]) that

\footnotetext{
${ }^{1}$ In this respect, one can remark that the idea behind surrogates differs from other resampling plans such as the bootstrap since randomization is not obtained by shuffling in some way actual values of the data but by generating new values in a completely independent manner.
} 
incorporates the observation scale. In this respect, strict stationarity may appear as a too strong commitment, and having access to a softened version could prove useful.

Whereas we could imagine to relax the usual i.i.d. hypothesis by replacing the actual spectrum phase by some correlated sequence, we choose here to rather modify the original phase according to:

$$
\Psi_{X}[f] \rightarrow \Psi_{S}[f]:=\Psi_{X}[f]+\Theta[f],
$$

where $\Theta[f]$ stands for some random phase noise.

It follows from eqs.(1) and (2) that the frequency-frequency distribution function of the so-obtained surrogates $s[n]$ (i.e., the 2D Fourier transform of the corresponding covariance function $\mathbb{E}\left\{s[n] s\left[n^{\prime}\right]\right\}$ in the time domain) reads

$$
\Phi_{S}\left[f, f^{\prime}\right]=\Phi_{X}\left[f, f^{\prime}\right] . \Lambda\left[f, f^{\prime}\right],
$$

with

$$
\Phi_{X}\left[f, f^{\prime}\right]:=A_{X}[f] A_{X}\left[f^{\prime}\right] \exp \left\{i\left(\Psi_{X}[f]-\Psi_{X}\left[f^{\prime}\right]\right)\right\}
$$

and

$$
\Lambda\left[f, f^{\prime}\right]:=\mathbb{E}\left\{\exp \left\{i\left(\Theta[f]-\Theta\left[f^{\prime}\right]\right)\right\}\right\} .
$$

In such a picture, stationarization corresponds to the situation where this term forces the spectral distribution function to only exist on the main diagonal of the plane, i.e., $\Lambda\left[f, f^{\prime}\right]=\delta\left[f-f^{\prime}\right]$.

Within this perspective, different options are offered, that may lead to new versatile forms of surrogate data. Depending on the type and level of the added phase noise $\Theta[f]$, the rationale of such a perturbation is therefore to allow for a transition between the original signal and its classical surrogates, whence the name "transitional surrogates".

\subsection{WGN transitional surrogates}

The first possibility is to choose for $\Theta[f]$ a white Gaussian noise (WGN), i.e., an i.i.d. Gaussian sequence of variance $\sigma^{2}$. It directly follows from the assumed Gaussianity that the correcting factor (4) to $\Phi_{X}\left[f, f^{\prime}\right]$ that appears in eq.(3) expresses as:

$$
\Lambda\left[f, f^{\prime}\right]=\exp \left\{-\frac{1}{2} \mathbb{E}\left\{\left(\Theta[f]-\Theta\left[f^{\prime}\right]\right)^{2}\right\}\right\},
$$

a quantity that can be equivalently rewritten as

$$
\Lambda\left[f, f^{\prime}\right]=\exp \left\{-c_{\Theta}[0]\right\} \cdot \exp \left\{c_{\Theta}\left[f-f^{\prime}\right]\right\}
$$

if we furthermore assume the sequence $\Theta[f]$ to be zero-mean and stationary, with correlation function $c_{\Theta}[$.$] (and therefore$ such that $c_{\Theta}[0]=\sigma^{2}$ ). This is indeed the case with the chosen i.i.d. model for which we have:

$$
c_{\Theta}\left[f-f^{\prime}\right]=\sigma^{2} \delta\left[f-f^{\prime}\right],
$$

leading to

$$
\Lambda\left[f, f^{\prime}\right]=\exp \left\{\sigma^{2}\left(\delta\left[f-f^{\prime}\right]-1\right)\right\} .
$$

If we only consider the main diagonal $f=f^{\prime}$ of the frequency-frequency plane, it thus happens that we always have $\Lambda\left[f, f^{\prime}\right]=1$ whereas, for any other values $f \neq f^{\prime}$, we have $\Lambda\left[f, f^{\prime}\right]=\exp \left\{-\sigma^{2}\right\}$. As expected, varying $\sigma$ from zero to infinity allows therefore to gradually move from the possibly nonstationary situation attached to $\Phi_{X}\left[f, f^{\prime}\right]$ to a function $\Phi_{S}\left[f, f^{\prime}\right]$ that is diagonal since

$$
\lim _{\sigma^{2} \rightarrow \infty} \Lambda\left[f, f^{\prime}\right]=\delta\left[f-f^{\prime}\right]
$$

and, hence, to a (second order) stationarized situation. All intermediate situations of such "WGN transitional surrogates" (WGNTS) correspond to a transition between these two extremes.

Remark - The choice made above to add a stationary Gaussian sequence to the phase is not the only one for obtaining transitional surrogates. It must however be remarked that, when adding a stationary sequence, departing from the uniform distribution attached to classical surrogates is instrumental in order to guarantee a transition. Indeed, it is possible to rephrase the above derivation starting from eq.(4) as follows. If we assume that $\Theta[f]$ is some i.i.d. sequence of characteristic function $\Psi_{\Theta}(u)$, the correcting factor (4) simply happens to be

$$
\Lambda\left[f, f^{\prime}\right]= \begin{cases}\left|\Psi_{\Theta}(1)\right|^{2} & \text { if } f \neq f^{\prime} \\ 1 & \text { if } f=f^{\prime}\end{cases}
$$

Assuming that $\Theta[f] \sim \mathcal{N}\left(0, \sigma^{2}\right)$, we readily get that $\Psi_{\Theta}(u)=$ $\exp \left\{-\sigma^{2} u^{2} / 2\right\}$ and thus recover the previously obtained result (6). This has however to be contrasted with the situation where the phase would be uniformly distributed as $\Theta[f] \sim \mathcal{U}(-\pi, \pi))$. In this case, we would have $\boldsymbol{\Psi}_{\Theta}(u)=$ $\sin \pi u / \pi u$, thus leading to $\boldsymbol{\Psi}_{\Theta}(1)=0$, and hence to exact stationarity.

\subsection{Wiener-Lévy transitional surrogates}

Turning back to (4), it appears that the correcting factor can also be expressed via a characteristic function, namely

$$
\Lambda\left[f, f^{\prime}\right]=\Psi_{\Delta \Theta}(1)
$$

where $\Delta \Theta\left[f, f^{\prime}\right]:=\Theta[f]-\Theta\left[f^{\prime}\right]$ stands for the increment process of the phase. Since the objective is to get a factor which is a weighting function depending only on the frequency difference $\left|f-f^{\prime}\right|$ (so as to ultimately converge to $\delta\left[f-f^{\prime}\right]$ ), a second possibility is to consider for the added phase a process with stationary increments, in which case it follows from (5) that

$$
\Lambda\left[f, f^{\prime}\right]=\exp \left\{-\frac{1}{2} \rho_{\Theta}\left[f-f^{\prime}\right]\right\},
$$

where $\rho_{\Theta}[$.$] stands for the second-order structure function [4]$ (or variogram [5]) of the added phase. This can be achieved with simple models of random walks and generalizations thereof, such as fractional Gaussian noises [6] for which $\rho_{\Theta}\left[f-f^{\prime}\right]=\left(\sigma^{2} / 2\right)\left|f-f^{\prime}\right|^{2 H}$, with $0<H<1$. Within the framework of such a model, we simply get

$$
\Lambda\left[f, f^{\prime}\right]=\exp \left\{-\frac{\sigma^{2}}{2}\left|f-f^{\prime}\right|^{2 H}\right\},
$$




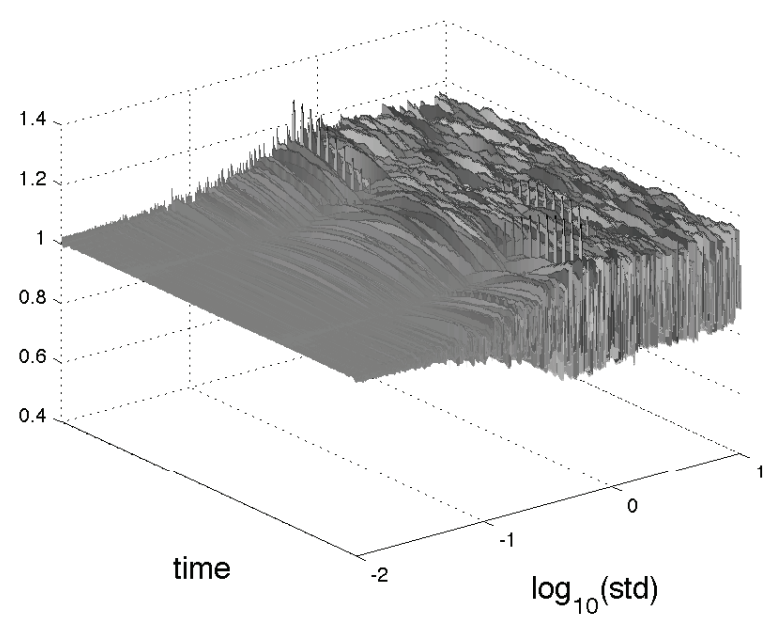

Fig. 1. Time envelopes of white Gaussian noise transitional surrogates (WGNTS) in the case of a unimodular pure tone, as a function of the added phase noise standard deviation $\sigma$.

with the expected (transitional and limiting) behaviour as a function of both the added noise standard deviation $\sigma$ and the Hurst exponent $H$ that controls the increment correlation. Whereas making use of both degrees of freedom could be interesting, we will restrict here to the only uncorrelated case $H=1 / 2$ corresponding to a standard Wiener-Lévy process for $\Theta[f]$, the associated surrogates being referred to as "Wiener-Lévy transitional surrogates" (WLTS).

\subsection{Examples}

In order to first illustrate in a qualitative way the behaviour of transitional surrogates, Fig. 1 displays how the envelope of a unimodular pure tone gradually moves from unity to a more erratic shape when the level of the added random phase (WGN in this specific case) is increased. As expected, an effective transition is indeed observed for such WGNTSs (a similar result would have been obtained with WLTSs, up to some possibly different range of $\sigma$ for the region where the most dramatic change occurs). In this case where surrogates are aimed at preserving stationarity in a supposed "stationary" situation, the benefit that can be gained from transitional surrogates is intuitively clear, with moderate levels of noise avoiding to directly switch from the unimodular tone to the "classical" surrogates (obtained in the limit $\sigma \rightarrow \infty$ ) for which a highly fluctuating envelope would be obtained.

As a companion example, Fig. 2 refers to a nonstationary test signal, namely the AM-FM example considered in Fig. 1 of [2]. In this case, the representation space is chosen as the $(P, F)$ plane, where $P$ and $F$ stand for the standard deviations of the instantaneous power and of the instantaneous frequency, obtained respectively as the zero-th and first order local moments of a time-frequency distribution. For each value of the noise level $\sigma$ and according to the principle of the one-class classifier described in [2], $(P, F)$ values of a given number of surrogates (typically 50 , as in [2]) are displayed

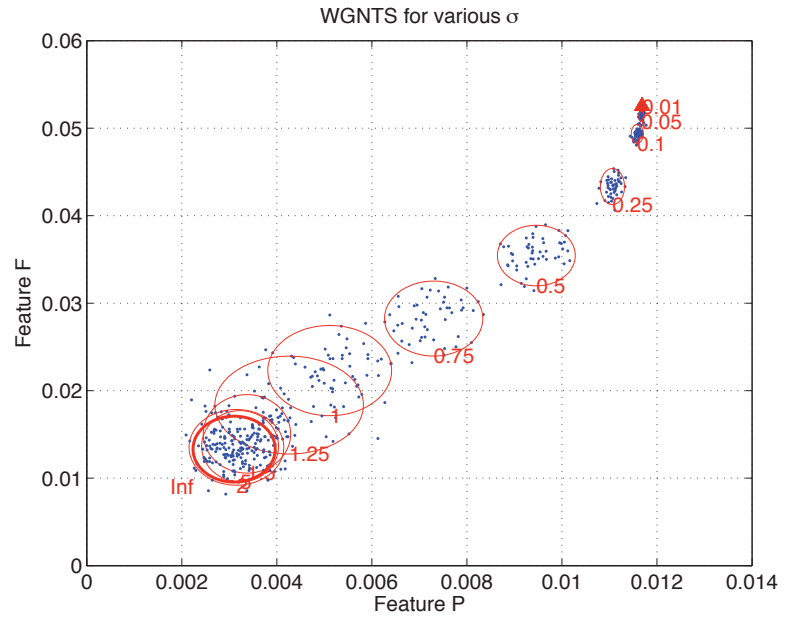

Fig. 2. WGNTS migration in the $(P, F)$ plane, red ellipses corresponding to different levels of the added noise (see text).

as blue dots, together with the (red) ellipse in which most of them are contained. More precisely, each elliptic domain (associated to the value of $\sigma$ written nearby) is chosen so as to contain a percentage equal to $100-\nu$ of the corresponding surrogates (in the case of Fig. 2, $\nu=15 \%$ ), and the detection strategy amounts to consider the analyzed signal as stationary if its $(P, F)$ value falls inside the elliptic domain, and nonstationary otherwise. Since the $(P, F)$ value of the actual signal is the one located on the top right of the diagram (red triangle), and since the stationary domain attached to the classical surrogates corresponds to the most left ellipse, Fig. 2 clearly evidences how increasing the noise level ends up with a transition that makes progressively the surrogates domain more and more distant from the observation, thus revealing more and more clearly the nonstationarity of the latter.

\subsection{Improving stationarity tests}

So as to give a quantitative appreciation of what happens when using transitional surrogates in the context of stationarity testing, two families of results will now be presented.

Under $\mathrm{HO}$ - The first one concerns the ability to reproduce the null hypothesis $\mathrm{H} 0$ of stationarity, i.e., to give an effective false alarm rate (as measured by the empirical percentage of surrogates features falling outside the stationarity ellipse) which agrees with the prescribed level of confidence $\nu$. This is reported in Fig. 3, as a function of the standard deviation of the added phase noise, in both cases of WGNTSs and WLTSs, and for 3 values of $\nu$. The simulations have been conducted with 1,000 realizations of an $\mathrm{AR}(2)$ process as test signal, leading to the $95 \%$ coverages displayed as shaded areas. For each realization, 50 surrogates have been generated and used to compute the elliptic domains associated to the null hypothesis of stationarity with the prescribed level of confidence. This domain is then used to test the stationarity of the current AR signal. The overall result is that a high level of noise (with the limiting case of standard surrogates displayed at the 


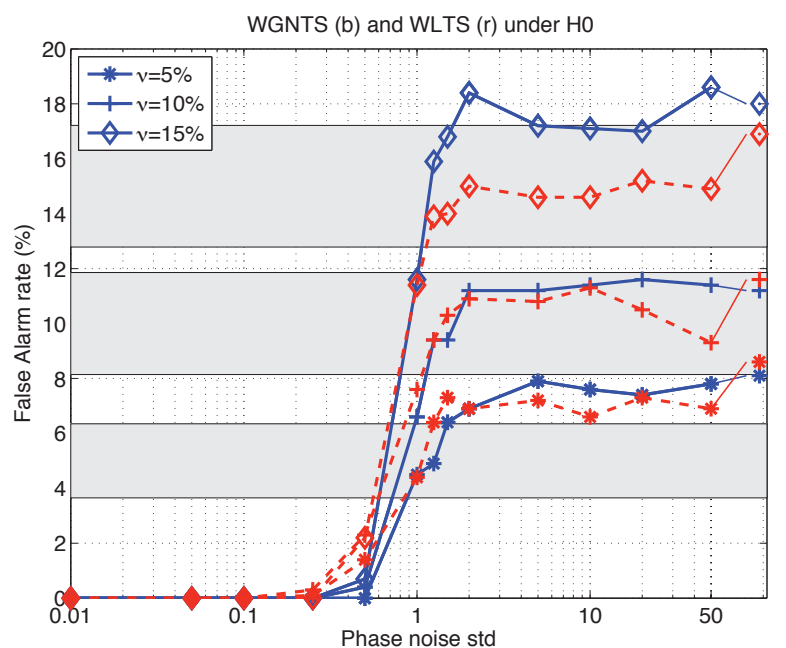

Fig. 3. Reproduction of the null hypothesis of stationarity for various confidence levels $\nu$ (see legend) and the two types of transitional surrogates (WGNTSs in solid blue and WLTSs in dashed red).

extreme right of the diagram) tends to make the test slightly pessimistic, while the limit $\sigma \rightarrow 0$ leads of course to a degenerate situation since all surrogates tend to identify to the actual test signal. In between these two extremes, it turns out that the performance can be uniformly improved (w.r.t. to both $\nu$ and the type of transitional surrogates) by choosing $\sigma \approx 1$.

Under H1 - Inspired by the examples considered in [2], the second family of results deals with a signal whose degree of nonstationarity can be varied, namely

$$
x[n]=\left[1+m \cos \left(2 \pi n / N_{0}\right)\right] \cdot e[n], 1 \leq n \leq N,
$$

where $e[n]$ stands for standard (stationary) WGN, $0 \leq m \leq 1$ is the index of the amplitude modulation and the ratio between the observation length and the modulation period is fixed to $N / N_{0} \simeq 5$ so as to guarantee some easily observable nonstationarity when $m$ is large enough. Under this "H1" hypothesis, the probability of estimating the signal as stationary is estimated as before (except that simulations have been run on 200 realizations only for each point) and the result is plotted in Fig. 4 as a function of both the index $m$ and the standard deviation $\sigma$ of the added noise. ${ }^{2}$ What is observed is again a transition in the performance, with a collapse of the different curves onto the one obtained with "classical" surrogates when $\sigma$ exceeds, as was the case under $\mathrm{H} 0$, the critical value 1 .

It follows from Figs. 3 and 4 that, as expected, transitional surrogates end up with some transitions in performance, regarding both $\mathrm{HO}$ and $\mathrm{H} 1$. The interpretation is quite clear since, as illustrated in Fig. 2, varying the level of the added phase noise results in a moving of the stationarity ellipse in the feature plane, and thus the probability of falling within it is varied accordingly.

\footnotetext{
${ }^{2}$ Only the performance with WGNTSs is reported here for the sake of clarity and because of space limitation, but WLTSs lead to similar results.
}

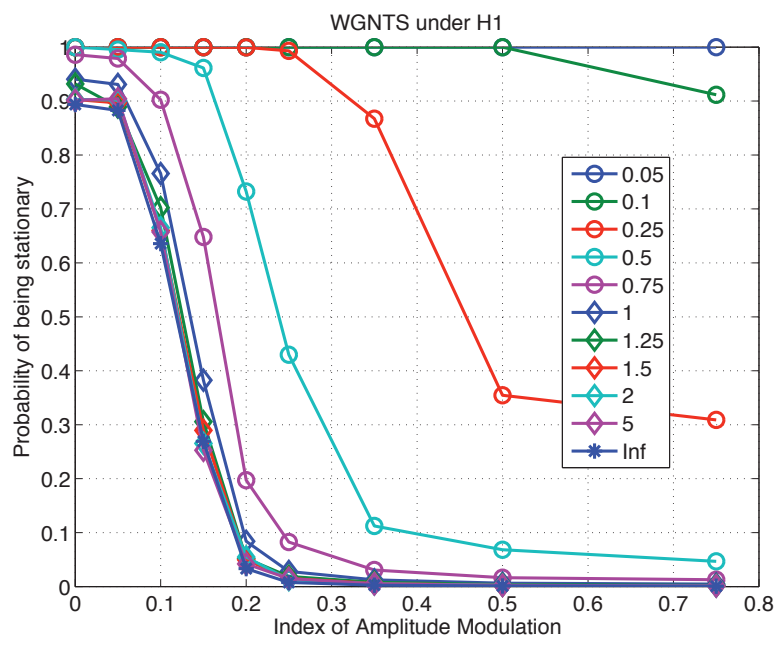

Fig. 4. Performance in nonstationarity detection for various standard deviations $\sigma$ (see legend) of the added phase noise and a confidence level $\nu=15 \%$.

\section{CONCLUSION}

A modified version of surrogates has been proposed, which offers the possibility of better tuning stationarity tests. In particular, it is worth noticing that, at least in the examples considered in Sect. 2.4, the false alarm rate can be set at the prescribed level without sacrificing detection. Not all the possibilities of the method have been explored here. In the WLTS case for instance, one could think of going beyond varying only the phase noise level by using simultaneouly different values of $H$ for controlling the correlation of the phase sequence. Alternatively, one can also consider a distribution of $\sigma$ 's in order to enlarge the stationarity domain. This is under current investigation.

Acknowledgments - This work is part of the ANR Project "StaRAC", supported by Grant ANR-07-BLAN-0191.

\section{REFERENCES}

[1] J. Theiler, S. Eubank, A. Longtin, B. Galdrikian, and J. Doyne Farmer, "Testing for nonlinearity in time series: the method of surrogate data," Physica D: Nonlinear Phenomena, vol. 58, no. 1-4, pp. 77-94, 1992.

[2] P. Borgnat, P. Flandrin, P. Honeine, C. Richard, and J. Xiao, "Testing stationarity with surrogates: A time-frequency approach," IEEE Trans. Signal Processing, vol. 58, no. 7, pp. 3459-3470, 2010.

[3] C. Richard, A. Ferrari, H. Amoud, P. Honeine, P. Flandrin, and P. Borgnat, "Statistical hypothesis testing with time-frequency surrogates to check signal stationarity," in Proc. IEEE International Conference on Acoustics, Speech and Signal Processing, Dallas, TX, USA, March 2010.

[4] A.M. Yaglom, Correlation Theory of Stationary and Related Random Functions I., Springer Verlag, 1986.

[5] N. Cressie, Statistics for Spatial Data, Wiley Interscience, 1993.

[6] P. Embrechts and M. Maejima, Selfsimilar Processes, Princeton Univ. Press, 2002. 